\title{
Editorische Hinweise
}

Der vorliegende thematische Band enthält Engels' Werk "Herrn Eugen Dührings Umwälzung der Wissenschaft (Anti-Dühring)" sowie die von Engels dazu angefertigten Vorarbeiten und die Vorarbeiten von Marx zum zweiten Abschnitt des "Anti-Dühring". Der Band enthält ferner die Schrift "Socialisme utopique et socialisme scientifique" (einschließlich des "Avant-propos" von Marx) sowie deren deutsche Fassung "Die Entwicklung des Sozialismus von der Utopie zur Wissenschaft" (mit dem Anhang "Die Mark"), da diese beiden Schriften unmittelbar auf der Grundlage des "Anti-Dühring" entstanden sind und die deutsche Fassung ihrerseits wesentlich auf die weiteren Auflagen des "Anti-Dühring" zurückwirkte. Beide Fassungen bildete die Grundlage für Übersetzungen, von denen die durch Engels autorisierten in die entsprechenden chronologischen Bände aufgenommen werden.

Der Band beginnt mit Engels' "Vorarbeiten zum ,Anti-Dühring'“. Sie bestehen aus den kommertierten Auszügen „E. Dühring: Kursus der National- und Sozialökonomie einschließlich der Hauptpunkte der Finanzpolitik. 2. Aufl. Leipzig 1876", den "Notizen für den ,Anti-Dühring'", dem Text "Der moderne Sozialismus" - dem Entwurf der Einleitung zum Gesamtwerk -, der Ausarbeitung "Taktik der Infanterie aus den materiellen Ursachen abgeleitet. 1700-1870", den "Noten zum ,Anti-Dühring' " und dem Entwurf "Alte Vorrede zum ,Anti-Dühring'. Über Dialektik". Aufgenommen werden alle Texte, die nachweislich für den "Anti-Dühring" angefertigt wurden, auch wenn sie wie die in den "Notizen für den ,Anti-Dühring'" enthaltene Ausarbeitung [3] mit den Texten "Die ewigen Naturgesetze" und "Sklaverei" sowie dem ersten Teil der Ausarbeitung "Der moderne Sozialismus. Entwurf der Einleitung zum ,Anti-Dühring", wie die "Noten zum ,Anti-Dühring'" und die "Alte Vor- 
rede zum ,Anti-Dühring'. Über Dialektik" von Engels später der "Dialektik der Natur" zugeordnet wurden.

Die Auszüge und Kommentare zu Dührings "Cursus der National- und Socialökonomie einschliesslich der Hauptpunkte der Finanzpolitik", bislang als zweiter Teil der Vorarbeiten ediert, werden entsprechend ihrer Entstehungszeit an die Spitze der Vorarbeiten gestellt. Die innerhalb dieser Ausarbeitung überlieferten Texte [9] "Dühring, Sozialismus." und [11] "Theilung der Arbeit" werden in die "Notizen für den ,Anti-Dühring'" eingefügt.

Bei den "Notizen für den "Anti-Dühring'" werden die einzelnen Bogen bzw. Blätter, die als Texteinheiten jeweils eine redaktionelle Num. mer erhalten, chronologisch angeordnet. Die auf den einzelnen Bogen bzw. Blättern sich befindenden Texte werden geschlossen veröffentlicht, auch wenn sie zu verschiedenen Zeiten niedergeschrieben wurden. Die chronologische Anordnung erfolgt nach der ersten Notiz auf dem jeweiligen Bogen bzw. Blatt. Anhaltspunkte für die Anordnung bilden Inhalt und Charakter der einzelnen Texte.

Die Vorarbeit "Der moderne Sozialismus. Entwurf der Einleitung zum ,Anti-Dühring' " wird unabhängig von der konkreten Überlieferungslage geschlossen als selbständiger Text dargeboten.

Die Begründung für die Datierung und Anordnung der "Notizen ..." sowie der anderen Vorarbeiten wird im jeweiligen Apparatteil Entstehung und Überlieferung gegeben.

Als geschlossener Komplex werden in der Reihenfolge ihres Entstehens Marx' "Vorarbeiten zum zweiten Abschnitt des "Anti-Dühring'" veröffentlicht. Dieser Teil enthält die "Randnoten zu Dührings ,Kritischer Geschichte der Nationalökonomie' ", den dazu angefertigten Entwurf (als erste vollständige Veröffentlichung), die im Zusammenhang damit entstandenen Notizen (als Erstveröffentlichung) sowie die Ausarbeitung "Das Tableau économique mit einigen Randglossen". Erstmals vollständig veröffentlicht werden die "Auszüge aus Smith und Rodbertus über Einkommensarten und Besitzrente".

Die Grundlage für den Edierten Text des Werkes "Herrn Eugen Dührings Umwälzung der Wissenschaft (Anti-Dühring)" bildet die erste Buchausgabe (1878). Die abweichenden Stellen in den anderen überlieferten Textzeugen werden im Variantenverzeichnis wiedergegeben.

Gesondert werden im Edierten Text das Kapitel X "Aus der ,Kritischen Geschichte" " des zweiten Abschnitts und das Kapitel II "Theoretisches" des dritten Abschnitts in der Fassung der dritten Auflage des "Anti-Düh. ring" dargeboten, da sie gegenüber der ersten Auflage stark überarbeitet und erweitert wurden. Dem Charakter eines thematischen Bandes 
Rechnung tragend, werden diesem Komplex die Vorworte zu den drei Auflagen des "Anti-Dühring" in der Fassung der dritten Auflage vorangestellt.

Dem Edierten Text von Engels' Schrift "Socialisme utopique et socialisme scientifique" wird der Separatabdruck zugrunde gelegt. Dem Text der Broschüre vorangestellt wird Marx' "Avant-propos" und dessen von Paul Lafargue redigierte Fassung.

Der Anhang "Die Mark" zu Engels' "Entwicklung des Sozialismus von der Utopie zur Wissenschaft" wird im Rahmen dieser Schrift veröffentlicht. Er enthält jedoch einen eigenen Apparat, da er in Entstehung, Überlieferung und Wirkung eine relative Eigenständigkeit aufweist.

Die Begründung für die Wahl der Textgrundlage ist den Hinweisen zur Edition der jeweiligen Arbeiten zu entnehmen.

Der Edierte Text folgt der festgelegten Textgrundlage. Neu entziffert wurden die bisher nicht veröffentlichten Texte und die Mehrzahl der Varianten.

Eine Vereinheitlichung oder Modernisierung der Orthographie und Interpunktion wird nicht vorgenommen, jedoch erfolgt eine Textrevision zur Beseitigung eindeutig fehlerhafter Textstellen.

Eindeutige Schreib- und Druckfehler werden im Edierten Text korrigiert und nicht in das Korrekturenverzeichnis aufgenommen. Darunter fallen auch Druckfehler, die aufgrund von Autorkorrekturen in Wiederveröffentlichungen und Druckfehlerberichtigungen ermittelt werden konnten. Sinnverändernde redaktionelle Korrekturen werden stets im Korrekturenverzeichnis ausgewiesen. Schreib- und Druckfehler, deren Korrektur in verschiedener Weise möglich ist bzw. die nicht eindeutig als solche zu bestimmen sind, werden im Falle einer hinreichenden Begründung im Edierten Text berichtigt, in unklaren Fällen jedoch nicht bereinigt. Entsprechende Hinweise enthalten die Korrekturenverzeichnisse.

Versehen bei Faktenangaben sowie bei der Schreibweise von Namen, soweit sie eindeutig als solche bestimmbar sind, werden im Edierten Text korrigiert. Diese Berichtigungen sind im Korrekturenverzeichnis ausgewiesen. Ist der Sachverhalt nicht eindeutig, wird keine Veränderung vorgenommen, aber in den Erläuterungen darauf verwiesen.

Nichi mit Sicherheit entzifferbare Buchstaben in Handschriften werden in kleinerem Druck, unleserliche Buchstaben durch $x$ wiedergegeben. Textverluste, die durch Beschädigung oder Verschmutzung des $\mathrm{Pa}$ piers entstanden sind, werden im Edierten Text rekonstruiert und in eckigen Klammern eingefügt. Gleiches gilt für die Darbietung der durch 
Beschneiden entstandenen Textverluste bei den Randbemerkungen in Engels' Handexemplaren von Dührings "Cursus und National- und Socialökonomie ..." und "Kritischer Geschichte ..." in den Erläuterungen.

Die Interpunktion der zugrunde gelegten Handschriften bzw. des Druckes wird beibehalten. Nur offensichtliche Interpunktionsfehler werden im Text ohne Kennzeichnung korrigiert, soweit dadurch keine Sinnänderung eintritt. Besonderheiten bei den Vorarbeiten von Marx und Engels werden in den Hinweisen zur Edition zu den jeweiligen Texten erläutert. Anführungszeichen sowie halbe Anführungszeichen werden in einheitlicher Weise gesetzt, auch wenn dies von der jeweiligen Textgrundlage abweicht.

Abkürzungen werden nur dann ausgeschrieben, wenn sie ungebräuchlich sind; kann dies nicht eindeutig vorgenommen werden, erfolgt die Ausschreibung in eckigen Klammern. Die für Engels' Handschriften typischen Abkürzungen, Wortverkürzungen und Kurzzeichen wurden in den Hinweisen zur Edition näher beschrieben. In bibliographischen Angaben bleiben Abkürzungen von Personennamen und übliche Abkürzungen (Zitate und Literaturhinweise von Marx und Engels) bestehen.

Die verschiedenen Hervorhebungsstufen in handschriftlichen und gedruckten Textvorlagen werden im Edierten Text einheitlich folgendermaßen wiedergegeben: erste Hervorhebungsstufe - kursiv; zweite Hervorhebungsstufe - gesperrt; dritte Hervorhebungsstufe - kursiv gesperrt. Das Schrift- bzw. Druckbild des zugrunde gelegten Zeugen (Schriftart, Schriftgröße usw.) bleibt unberücksichtigt. Alle hierzu erforderlichen Angaben bietet die Zeugenbeschreibung.

Verwendungsvermerke und Merkzeichen, die Marx und Engels am Rand anbrachten, werden im Edierten Text und im Apparat in stilisierter Form reproduziert.

Beginn und Ende einer Seite der handschriftlichen bzw. gedruckten Textvorlage werden im Edierten Text kenntlich gemacht, und die Paginierung wird - wenn vorhanden - mitgeteilt (siehe S. 656). Wurde die Paginierung bei Handschriften redaktionell ergänzt oder verändert, so wird die Seitenzahl in eckige Klammern gesetzt.

Über Besonderheiten der Wiedergabe der handschriftlichen Textgrundlage von Marx und Engels geben die Hinweise zur Edition der jeweiligen Texte Auskunft.

$\mathrm{Zu}$ jeder in den Band aufgenommenen Arbeit wird ein wissenschaftlicher Apparat geboten. Er besteht aus dem Teil Entstehung und Überlieferung (einschließlich Zeugenbeschreibung und Begründung des edito- 
rischen Verfahrens), dem Variantenverzeichnis, dem Korrekturenverzeichnis und den Erläuterungen (siehe MEGA ${ }^{(2)}$ 1/1. S. 46*-50*). In der Kopfleiste werden die Entstehungszeiten sowie die Seitenzahlen des betreffenden Edierten Textes mitgeteilt.

Dem wissenschaftlichen Apparat des Bandes wird eine allgemeine Textgeschichte zu Engels' Arbeit am „Anti-Dühring“ vorangestellt. Der Apparat zu Marx” Vorarbeiten für den zweiten Abschnitt des „Anti-Dühring" beginnt ebenfalls mit einer allgemeinen Textgeschichte.

Der wissenschaftliche Apparat zu jeder einzelnen Arbeit beginnt mit der Darlegung ihrer Entstehung und Überlieferung; von der jeweiligen Wirkungsgeschichte wird nur das unmittelbare zeitgenössische Echo erfaßt.

Bei Handschriften, die zu Lebzeiten von Marx und Engels nicht veröffentlicht wurden, wird auf ihre Erstveröffentlichung hingewiesen.

In den Zeugenbeschreibungen werden alle für die Textentwicklung belangvollen Zeugen mit einer Sigle versehen (siehe S.653-656) und zusätzlich mit Zahlenexponenten bezeichnet. Diese Numerierung erfolgt unabhängig vom Charakter des einzelnen Zeugen fortlaufend in der Reihenfolge ihrer Entstehung (z. B. J', D2, D ${ }^{3}$ ). Nicht autorisierte Zeugen werden mit kleinen Buchstaben bezeichnet.

Das Variantenverzeichnis enthält alle von Marx und Engels vorgenommenen Textänderungen, die den Text inhaltlich oder stilistisch weiterentwickeln. Diese Varianten treten auf als Textreduzierungen (Tilgung nicht korrupter Textstellen), Textergänzungen (Einfügungen und Zusätze), Textersetzungen und Textumstellungen. Demzufolge werden folgende Textänderungen nicht verzeichnet: von Marx und Engels korrigierte Schreibfehler; von Marx und Engels vorgenommene Veränderun. gen der Orthographie oder Interpunktion, die keinen Einfluß auf die Sinngebung haben; Schreibansätze, die keinen erkennbaren Sinn ergeben oder bei denen der Sinn der ursprünglich vom Autor beabsichtig. ten Aussage nicht wenigstens mit Wahrscheinlichkeit rekonstruiert werden kann; innerhandschriftliche Sofortkorrekturen bei Zitaten aus Büchern anderer Autoren und solche, die formale Berichtigungen grammatischer oder stilistischer Versehen darstellen, jedoch weder die inhaltliche Aussage des Textes verändern noch den Stil der gesamten Darstellung wesentlich modifizieren.

Das Variantenverzeichnis ist ein mit notwendigen Stützworten aus dem Edierten Text versehener Werkstellenapparat, d.h., es verzeichnet von Werkstelle zu Werkstelle fortschreitend alle varianten Fassungen einer Textstelle. Die innerhandschriftlichen Varianten zu einer Werk. stelle werden entweder mit Hilfe diakritischer Zeichen hintereinander 
oder mit der Methode der Zeilenparallelisierung bzw. der Zeilengrup. penparallelisierung untereinander dargeboten. Das Variantenverzeich. nis benutzt eine im wesentlichen diskursive (schlußfolgernde) Verzeich. nungsform, d. h., es wird der Inhalt der Textänderungen festgehalten, jedoch nicht die Form, in der diese Änderungen durchgeführt wurden. Sind die Art bzw. die Reihenfolge der Textänderungen nicht eindeutig zu bestimmen, wird der handschriftliche Befund beschrieben oder direkt dargeboten. Das konkrete Verfahren wird in den Hinweisen zur Edition bei den jeweiligen Texten erläutert.

Textreduzierungen, Textergänzungen, Textersetzungen und Textumstellungen werden mit Hilfe verschiedener diakritischer Zeichen dargestellt (siehe Verzeichnis der Abkürzungen, Siglen und Zeichen). Sofortvarianten treten häufig auch in Form von Abbrechungen auf. Als Abbrechungen werden solche Textänderungen bezeichnet, bei denen der Autor die Gedankenführung unterbricht und ihr (meist durch Tilgung, aber auch durch Ersetzung von Wörtern oder Wortteilen, Änderung von Flexionsendungen und Einfügungen) einen neuen Verlauf gibt. Abbrechungen, die in der Handschrift getilgt wurden, werden folgendermaßen dargestellt: Nach dem Stützwort aus dem Edierten Text folgt in Winkelklammern der getilgte Passus und danach das Abbrechungszeichen. Die neue Version der Fortsetzung dieses Satzes ist im Edierten Text nachzulesen. Abbrechungen, bei denen Teile des Wortbestandes in die nächste Schicht übernommen wurden, werden im Prinzip ebenso dargestellt. $\mathrm{Da}$ in diesen Fällen oft nicht sicher zu erkennen ist, an welcher Stelle des Satzes der Autor abbrach und änderte, wird das Abbrechungszeichen in der Regel an die Stelle gesetzt, an der spätestens die Textumformung erfolgt sein muß; der in Winkelklammern stehende Text des ursprünglichen, abgebrochenen Satzverlaufs umfaßt in diesen Fällen also auch Wörter oder Wortteile, die in der Handschrift nicht getilgt, sondern in die neue Textfassung einbezogen wurden. Die Winkelklammern kennzeichnen hier ein im Ganzen verworfenes Textstück.

Einige Textumformungen, vor allem größere Textersetzungen, werden mit Hilfe der Zeilenparallelisierung dargestellt. Dabei werden Va. rianten einer Werkstelle in chronologischer Folge partiturähnlich untereinandergestellt, wobei jede Schicht, die links einen Zähler erhält, durch die nächstfolgende ersetzt wird. Die jeweils letzte Schicht ist identisch mit dem Edierten Text. Unverändert bleibende Wörter werden nicht wiederholt, sondern durch Unterführungszeichen gekennzeichnet. Der durchgehende Strich bezeichnet entweder eine Textreduzierung gegenüber der vorhergehenden Schicht oder ist nur ein Dehnungsstrich, um den Raum für eine Texterweiterung in der folgenden 
Schicht offenzuhalten. Man kann sowohl jede Schicht für sich im Zusammenhang (horizontal) lesen als auch die Entwicklung einzelner Werkstellen von Schicht zu Schicht (vertikal) überblicken. Partielle Textänderungen innerhalb einer Schicht werden durch Gabelungen dargestellt, die mit $\mathbf{a}, \mathbf{b}, \mathbf{c}$ usw. oder, wenn diese Veränderungen nochmals variieren, mit $\alpha, \beta, y$ usw. bezeichnet sind. Durch Parallelisierung werden auch kleinere Textveränderungen innerhalb größerer Textreduzierungen, -ersetzungen oder -erweiterungen dargestellt, da somit der Bereich der "inneren" Variante ohne zusätzliche Zeichen erkennbar ist.

Bei einigen umfangreichen Textveränderungen macht es sich erforderlich, die Zeilengruppenparallelisierung anzuwenden. Die Varianten einer Werkstelle werden in römisch bezifferten Zeilengruppen in chronologischer Folge untereinandergestellt; jede Gruppe wird durch die jeweils folgende aufgehoben. Die letzte Zeilengruppe (bzw. deren letzte Schicht) ist identisch mit dem Edierten Text.

Besonderheiten der Variantendarbietung in den Marxschen "Vorarbeiten zum zweiten Abschnitt des "Anti-Dühring' "werden in den entsprechenden Hinweisen zur Edition erläutert (siehe S. 854/855).

Die Erläuterungen geben alle für das Verständnis des Textes (einschließlich der Varianten) erforderlichen Erklärungen und Hinweise, soweit dies nicht schon im Apparatteil Entstehung und Überlieferung geschehen ist.

Wichtiger Bestandteil der Erläuterungen ist der Nachweis der von Marx und Engels benutzten Literatur. Wenn nicht ermittelt werden konnte, welche Ausgabe von innen benutzt wurde, bzw. die benutzte nicht verfügbar war, erfolgt in den Erläuterungen ein entsprechender Verweis. Abweichungen zwischen der Zitierweise der Autoren und der benutzten Quelle werden verzeichnet, wenn diese inhaltlich belangvoll oder für eine vorgenommene oder mögliche Textrevision von Bedeutung sind. Außerdem werden von Marx und Engels gegenüber der zitierten Quelle vorgenommene Hervorhebungen mitgeteilt. Bei Zitaten aus den Schriften Dührings stammen alle Hervorhebungen sowie die in runde Klammern gesetzten Einfügungen von Marx oder Engels; sie werden deshalb in den Erläuterungen nicht ausgewiesen. Bei indirekt erwähnter oder zitierter Literatur erfolgt - soweit nachweisbar - ein Hinweis auf die entsprechende Erstausgabe. Bei Zitaten aus der Weltliteratur wird in der Regel auf die Angabe einer konkreten Ausgabe verzichtet. Verweisungen auf die bisher erschienenen Bände aller Abteilungen der MEGA ${ }^{(2)}$ erfolgen unter Verwendung der im Verzeichnis der Abkürzungen entschlüsselten Siglen. In allen anderen Fällen wird bei $\mathrm{Zi}$ - 
taten aus Arbeiten von Marx und Engels direkt auf den Erstdruck oder das handschriftliche Manuskript verwiesen.

In den Erläuterungen wird des weiteren verwiesen von Textstellen aus den Engelsschen Vorarbeiten auf ihre Verwendung in der Druckfassung des "Anti-Dühring" bzw. von den entsprechenden Passagen der Druckfassung auf die Stellen in den Vorarbeiten, auf die sich Engels stützt.

Engels' Marginalien (Randbemerkungen, Merkzeichen, An- und Unterstreichungen) in seinen Handexemplaren der zweiten Auflage von Dührings "Cursus der National- und Socialökonomie ..." sowie der "Kritischen Geschichte ..." werden mitgeteilt, sofern sich Engels in den Vorarbeiten bzw. in der Druckfassung des "Anti-Dühring" auf die relevan. ten Stellen bezieht. Die Marginalien werden jeweils nur einmal beschrieben; bei mehrmaliger Verwendung wird auf die enstprechende Erläuterung verwiesen; Merkzeichen werden in allen Fällen mitgeteilt. Die Marginalien im Handexemplar der "Kritischen Geschichte ...", das von Karl-Ludwig König (Karl-Marx-Haus Trier) in der Bibliothek des Instituts für Sozialforschung in Frankfurt/Main aufgefunden wurde, als der wissenschaftliche Apparat dieses Bandes bereits im Umbruch vorlag, werden in dem gesonderten Apparatteil "Nachträge über die Marginalien im Handexemplar von Eugen Dührings ,Kritischer Geschichte der Nationalökonomie und des Socialismus, zweite, theilweise umgearbei. tete Auflage, Berlin 1875" "ausgewertet.

Die Register erfassen den Edierten Text und die Varianten.

Das Literaturregister umfaßt alle Literatur (Bücher, Broschüren, Zeitschriftenaufsätze, Zeitungsartikel usw.), die direkt oder indirekt zitiert, erwähnt oder benutzt wird. Die Titel anonymer Veröffentlichungen werden nach dem ersten Wort, das kein bestimmter oder unbestimmter Artikel ist, eingeordnet. Nicht aufgenommen werden allgemeine Hinweise auf Verträge, Verfassungen, Gesetze u. ä. sowie auf Manuskripte, Archivmaterialien und Briefe, die zum Zeitpunkt der Abfassung des Textes noch unveröffentlicht waren und es zum Teil auch heute noch sind.

Das Namenregister stellt alle direkt oder indirekt genannten Personennamen zusammen, wobei literarische und mythologische Namen einbezogen werden. Aufgenommen werden auch die Verfasser von Veröffentlichungen, die im Text selbst nicht genannt, deren Arbeiten aber direkt oder indirekt zitiert, erwähnt bzw. benutzt werden. Die alphabetische Einordnung der Personennamen erfolgt nach ihrer authentischen Schreibweise, bei griechisch oder kyrillisch geschriebenen $\mathrm{Na}$ men nach der entsprechenden transkribierten Form. Alle von der 
authentischen Form abweichenden Schreibweisen des Edierten Textes werden der authentischen Schreibweise in runden Klammern zugefügt und, wenn notwendig, gesondert als Verweisung angeführt.

Das Sachregister umfaßt die Begriffe, die den wesentlichen Inhalt der Arbeiten von Marx und Engels widerspiegeln. Die Schlagworte sind in der Regel dem Edierten Text entnommen oder lehnen sich weitgehend an diesen an. Das Sachregister ist in moderner Orthographie abgefaßt.

Der vorliegende Band wurde bearbeitet von Renate Merkel (Leitung), Karlheinz Geyer, Marianne Jentsch und Carl-Erich Vollgraf. Die Bearbeitung der "Mark" erfolgte durch Kurt Kozianka (Jena). Das Literaturregister wurde von Erika Bauke, das Namenregister von Karlheinz Geyer, das Sachregister von Marianne Jentsch und Carl-Erich Vollgraf zusammengestellt. Wissenschaftlich-technische Arbeiten führten Erika Bauke und Käte Schwank durch. Entzifferungen fertigten Jo Koch und Maria Lehmann an.

Der Band wurde seitens der Redaktionskommission betreut und begutachtet von Inge Taubert. Gutachter des IML beim ZK der KPdSU waren Georgi Bagaturija und Wassili Kusnezow.

Die Herausgeber danken allen wissenschaftlichen Einrichtungen und Personen, die bei der Vorbereitung des Bandes Unterstützung leisteten. Die Einsichtnahme in die Originale ermöglichte das Internationale Institut für Sozialgeschichte in Amsterdam. Das Institut für Sozialforschung in Frankfurt/Main gewährte Einsicht in das Handexemplar von Dührings "Kritischer Geschichte ...". Die Deutsche Staatsbibliothek Berlin ermöglichte die Durchsicht des Dühring-Nachlasses. Johannes Klare, Günter Kluge und Christian Wagner (Humboldt-Universität zu Berlin) fertigten die Analyse der französischen Übersetzung "Socialisme utopique ..." sowie die Verzeichnisse von Abweichungen an. Teilgutachten erfolgten durch Mitglieder des Wissenschaftlichen Rates für Marx-Engels-Forschung. Stellungnahmen bzw. Ausarbeitungen zu einzelnen Sachgebieten verfaßten Anneliese Griese (Berlin), Heinz Helmert (Leipzig), Ursula Herrmann (Berlin), Wolfgang Jahn (Halle), Liane Jauch (Berlin), Hansulrich Labuske (Berlin), Horst Schröder (Berlin) und Jutta Seidel (Leipzig). 
